\title{
El grado de cultura sobre responsabilidad social empresarial y la protección del medio ambiente en los estudiantes de Ingeniería Industrial, Sistemas e Informática
}

The grade of culture has more than enough managerial social responsibility and the protection of the environment in the students of Industrial Engineering, Systems and Computer science

Alfredo Edgar López Jiménez1', Víctor Fredy Espezua Serrano', Lino Rolando Rodríguez Alegre', William Fredy López Jiménez', Elver Stalin Ticliahuanca Calderón ${ }^{1}$

\section{RESUMEN}

Objetivo: Analizar el grado de cultura sobre responsabilidad social empresarial en los estudiantes de Ingeniería Industrial, Sistemas e Informática. Métodos: Se aplicó el método deductivo e inductivo. Se utilizó el modelo del diseño de investigación descriptivo correlacional. La población del estudio fue de 1200 estudiantes, del que se calculó un tamaño de muestra final de 235 estudiantes. Resultados: Los indicadores como negocios basados en valores, valores y principios éticos, y gestión responsable sugieren que los estudiantes poseen niveles intermedios de conocimientos, representando a la mayoría con un porcentaje del 59,6\%. La variable conciencia en protección del medio ambiente muestra que el $54,9 \%$ de los estudiantes son regulares. La pruebas de hipótesis para cada factor arrojaron una significación de muestra de $0,000(p<0,05)$ estableciendo una relación con las variables evaluadas en los estudiantes. Conclusiones: Si existe relación entre el Grado de Cultura sobre Responsabilidad Social Empresarial y la Protección del Medio Ambiente en los estudiantes de la Facultad de Ingeniería Industrial, Sistemas e Informática. Esta relación nos revela la importancia de la cultura de los estudiantes sobre la protección del medio ambiente, de tal manera que se contribuya en nuestra comunidad con el desarrollo sostenible de la economía local y nacional.

Palabras clave: Negocios, Valores, principios éticos, gestión responsable, responsabilidad social, protección del medio ambiente.

\section{ABSTRACT}

Objective: To analyze the culture grade has more than enough managerial social responsibility in the students of Industrial Engineering, Systems and Computer science. Methods: The deductive and inductive method was applied. The pattern of the design of investigation descriptive correlacional was used. The population of the study belonged to 1200 students, of which a size of 235 students' final sample was calculated. Results: The indicators as business based on values, values and ethical principles, and responsible administration suggest that the students possess intermediate levels of knowledge, representing most with a percentage of $59.6 \%$. The variable makes aware in protection of the environment it shows that $54.9 \%$ of the students is regular. The hypothesis tests for each factor threw a significance of sample of $0,000(p<0,05)$ establishing a relationship with the variables evaluated in the students. Conclusions: If relationship exists among the Grade of Culture it has more than enough Managerial Social Responsibility and the Protection of the Environment in the students of the Ability of Industrial Engineering, Systems and Computer science. This relationship reveals us the importance of the culture of the students on the protection of the environment, in such a way that is contributed in our community with the sustainable development of the local and national economy.

Keywords: Business, values, ethical principles, responsible administration, social responsibility, protection of the environment.

\footnotetext{
${ }^{1}$ Universidad Nacional José Faustino Sánchez Carrión, Huacho, Perú.
} 


\section{INTRODUCCIÓN}

En nuestro país la contaminación del medio ambiente, es ya, una preocupación, por ello nuestros ciudadanos comienzan cada vez más a tomar conciencia del cuidado o protección del medio ambiente. La toma de conciencia se viene promoviendo desde los menores de edad, y cuando lleguen a la adolescencia ya tienen que manifestar un compromiso definido. Sin embargo cuando los adolescentes llegan a las universidades a estudiar alguna carrera profesional, reciben un plan de estudios de diversos cursos de especialidad, con bajo énfasis en el cuidado y protección del medio ambiente.

Para Ortiz (2009), la responsabilidad social empresarial se origina desde una concepción de organización, que sustenta sus ideas, decisiones, acciones y resultados en valores, los cuales permiten la construcción de una sociedad con mayor riqueza pero de igual forma más justa, equitativa y sustentable. Esta filosofía se hace real cuando se realizan actividades formalizadas a través de las cuales se promueve el desarrollo de todos sus grupos, con o sin poder, mediante mayores oportunidades económicas, sociales, culturales, políticas y de sostenibilidad ecológica que le permite superar voluntariamente los niveles mínimos de exigencia: legal, ética y económica, y construir una sólida reputación que se sustenta en relaciones de confianza y le dan legitimidad en la sociedad en la que opera. Según Reyno (2006), las empresas deben planear conseguir un equilibrio entre sus resultados económicos y su intervención en la sociedad; la cual requiere un modelo de organización cuya misión, visión, estrategia y objetivos organizacionales estén directamente relacionados con el concepto de bienestar de la sociedad. Las organizaciones socialmente responsables son capaces de integrar su visión, misión, estrategia y objetivos organizacionales con un modelo de intervención social responsable. Su objetivo es alcanzar el equilibrio entre la responsabilidad social y su objetivo empresarial con el fin de obtener ventaja competitiva en el futuro. Las organizaciones deben tener en la cuenta que las prácticas socialmente responsables son una herramienta para que las grandes empresas la utilicen en función de crear una ventaja competitiva. Dichas prácticas deben integrarse a la estrategia de las organizaciones como una herramienta adicional creadora de ventajas competitivas. En la décima edición de la Expoferia de Proyectos de Responsabilidad Social Empresarial, que organizó la asociación civil Perú 2021, ABB (2016), compartió su experiencia, visión y sus planes de responsabilidad social. La Expoferia tuvo como objetivo promover y reconocer el comportamiento socialmente responsable de las empresas y aprovechar la ocasión para formar alianzas entre ellas. Inés Temple, presidenta de Perú 2021, afirmó que las empresas deben agregar valor a la sociedad en su conjunto y eso se muestra no tanto en lo que dicen de sí mismas, sino en lo que hacen. Sobre el compromiso por la educación, la Ing. Lourdes Cárdenas, Country Sustainability Controller de ABB (2016), indicó que la compañía busca difundir, ante la comunidad, su comportamiento ético y los valores que sostienen la relación con sus colaboradores y stakeholders. "Un ejemplo de ello son las acciones orientadas a formar personas más conscientes y responsables con el cuidado del medio ambiente y la salud". Destaco los proyectos de responsabilidad social en los que participó $A B B$ en Perú, entre ellos el de la promoción del Concurso de Aplicación de Eficiencia Energética, para la cual se asesoró, a más de 500 estudiantes de ingeniería de 15 universidades del país, en el uso responsable de la energía en la industria, motivándolos a presentar proyectos novedosos y eficientes para el sector empresarial, que es el gran responsable de consumo de la electricidad en el país.

Sobre la visión compartida del futuro, la responsabilidad social no es una iniciativa temporal, sino una política permanente que forma parte de la razón de ser de una empresa comprometida con el desarrollo de su comunidad. Así lo entiende el Ing. Diego Canta, Jefe de Seguridad, Salud Ocupacional y Medio Ambiente de ABB en Perú, quien afirma que la compañía pretende ser un agente de cambio en la sociedad. Explicó que la concepción de $A B B$ en Perú sobre la responsabilidad social es una visión de un futuro sostenible que comparte junto con sus 
clientes, quienes también encausan proyectos que van en la misma dirección, lo que demuestra que actividades como esta feria rinden frutos que nos devuelven la esperanza en la construcción de un país mejor.

En la formación de los futuros ingenieros industriales, y otros ingenieros, se requieren que interioricen los conceptos y pensamientos de responsabilidad social empresarial. Esta condición significa que los estudiantes de ingeniería deben de mostrar un cierto grado de cultura sobre la responsabilidad social y empresarial. Esta situación indica que los futuros ingenieros deben de recibir formación profesional moderna, en donde el concepto de productividad debe ir obligatoriamente acompañado del concepto de responsabilidad social.

Los profesionales del área de ingeniería, son los principales profesionales que pueden conducir a las empresas o negocios a tener un desarrollo sostenible con notoria responsabilidad social. Por esta razón es necesario evaluar los grados de conocimientos que van asimilando los estudiantes universitarios de ingeniería con respecto a la responsabilidad social empresarial.

En nuestra universidad, la formación de futuros profesionales de ingeniería pasa por un proceso de cambios de planes de estudios, lo cual podría estar variando la formación de conocimientos sobre la Responsabilidad Social Empresarial que deben de mostrar los estudiantes de ingeniería. Por ello, el objetivo de la presente investigación fue analizar la relación entre el nivel de conocimientos en responsabilidad social empresarial y la protección del medio ambiente en los estudiantes de ingeniería.

\section{MATERIALY MÉTODOS}

Nuestra investigación se desarrolló en los ambientes de la Universidad Nacional José Faustino Sánchez Carrión, la recolección de datos se realizó en los ambientes de las aulas de la Facultad de Ingeniería Industrial, Sistemas e Informática, con participación de los estudiantes de esta Facultad. Ellos constituyeron nuestra población cuya característica fue finita y que según los registros oficiales son 1200 estudiantes matriculados. El tamaño de muestra calculado fue de 235 estudiantes matriculados, se utilizó el Muestreo Simple o Aleatorio, el cual cumplió con la característica de ser probabilístico. Estos estudiantes fueron encuestadas al azar en el interior de las aulas de la Facultad en estudio. El Instrumento utilizado ha sido un modelo de encuesta elaborado por los propios autores, con cuestionario para medir los conocimientos sobre la responsabilidad social empresarial de los estudiantes, entre ellos; negocios basados en valores, valores y principios éticos, y gestión responsable y conciencia en protección del medio ambiente. Toda la información fue evaluada con el alpha de Cronbach.

\section{RESULTADOS}

El coeficiente alpha de Cronbach resulto con un valor del 0,741 , el cual lo ubica en una categoría de alta confiabilidad, así mismo tiene una validación de juicio de expertos del $93,3 \%$, que lo valida como muy bueno. La Tabla 1 muestra los resultados descriptivos de la investigación. En ella se consideraron los cinco indicadores evaluados en el presente estudio. 
Tabla 1. Resultados Descriptivos de la Investigación.

\begin{tabular}{lccc}
\hline \multicolumn{1}{c}{ Indicadores } & Categoría & Frecuencia & Porcentaje \\
\hline 1. Nivel de Conocimientos & Malo & 20 & $8,5 \%$ \\
sobre Negocios Basados en & Regular & 144 & $61,3 \%$ \\
Valores & Bueno & 71 & $30,2 \%$ \\
\hline \multirow{2}{*}{ 2. Conocimientos en Valores y } & Malo & 4 & $1,7 \%$ \\
Principios Éticos & Regular & 114 & $48,5 \%$ \\
& Bueno & 117 & $49,8 \%$ \\
\hline \multirow{2}{*}{ 3. Conocimientos en Gestión } & Malo & 32 & $13,6 \%$ \\
Responsable & Regular & 128 & $54,5 \%$ \\
& Bueno & 75 & $31,9 \%$ \\
\hline \multirow{2}{*}{$\begin{array}{l}\text { V1. Conocimientos en Gestión } \\
\text { Responsable }\end{array}$} & Malo & 12 & $5,1 \%$ \\
& Regular & 140 & $59,6 \%$ \\
\hline \multirow{2}{*}{ V2. Conciencia sobre } & Bueno & 83 & $35,3 \%$ \\
Protección del Medio Ambiente & Bajo & 12 & $5,1 \%$ \\
& Medio & 129 & $54,9 \%$ \\
\hline
\end{tabular}

El indicador nivel de conocimientos en negocios basados en valores visualiza que en primer lugar están los estudiantes cuyo conocimiento es regular, ellos representan a la mayoría con un porcentaje del $61,3 \%$, en segundo lugar se encuentra la categoría buena, representado por $30,2 \%$. Estos resultados nos expresan que los estudiantes de ingeniería tienen conocimientos medios sobre los negocios realizados con transparencias.

El indicador nivel de conocimientos en valores y principios éticos muestra en primer lugar a estudiantes cuyo conocimiento es bueno, representados con $49,8 \%$, en segundo lugar está la categoría regular, representado con $48,5 \%$. Estos resultados nos expresan que los estudiantes de ingeniería tienen conocimientos favorables sobre los valores y ética que debe aplicarse en los negocios en forma general.

El indicador nivel de conocimientos en gestión responsable muestra en primer lugar a estudiantes cuyo conocimiento es regular, representado por $54,5 \%$, en segundo lugar están los que pertenecen a la categoría buena, ellos están representados con un $31,9 \%$. Estos resultados nos expresan que los estudiantes de ingeniería tienen conocimientos intermedios sobre las acciones que involucran el mundo de la gestión responsable.

La variable nivel de responsabilidad social empresarial muestra en primer lugar a estudiantes cuyo conocimiento es regular, representados con el 59,6\%, en segundo lugar están los que pertenecen a la categoría buena, ellos están representados con un $35,3 \%$. Estos resultados nos expresan que los estudiantes de ingeniería tienen conocimientos intermedios sobre responsabilidad social alguna actividad de negocio con la formalidad que la ley de país establece.

En la variable nivel de conciencia de protección del medio ambiente se aprecia que en primer lugar están los estudiantes cuya conciencia es media, representados por el $54,9 \%$, en segundo lugar están los que tienen conciencia alta, representados con un $40,0 \%$. Estos resultados nos manifiestan que el nivel de conciencia sobre protección del medio ambiente que tienen los estudiantes de ingeniería, es intermedia, lo cual indica que se debe de fortalecer para mejorar la protección del medio ambiente. 
La Figura 1 y Tabla 2 muestra los resultados inferenciales de la investigación. En los contrastes conocimientos en negocios basados en valores y conciencia en protección del medio ambiente; conocimientos en valores y principios éticos y conciencia en protección del medio ambiente; conocimientos en gestión responsable y conciencia en protección del medio ambiente; conocimientos en responsabilidad social empresarial y conciencia en protección del medio ambiente, se obtuvieron una significancia de 0,000 ( $p<$ $0,05)$ lo que se establece una relación entre las variables evaluadas en los estudiantes de la Facultad de Ingeniería Industrial, Sistemas e Informática.

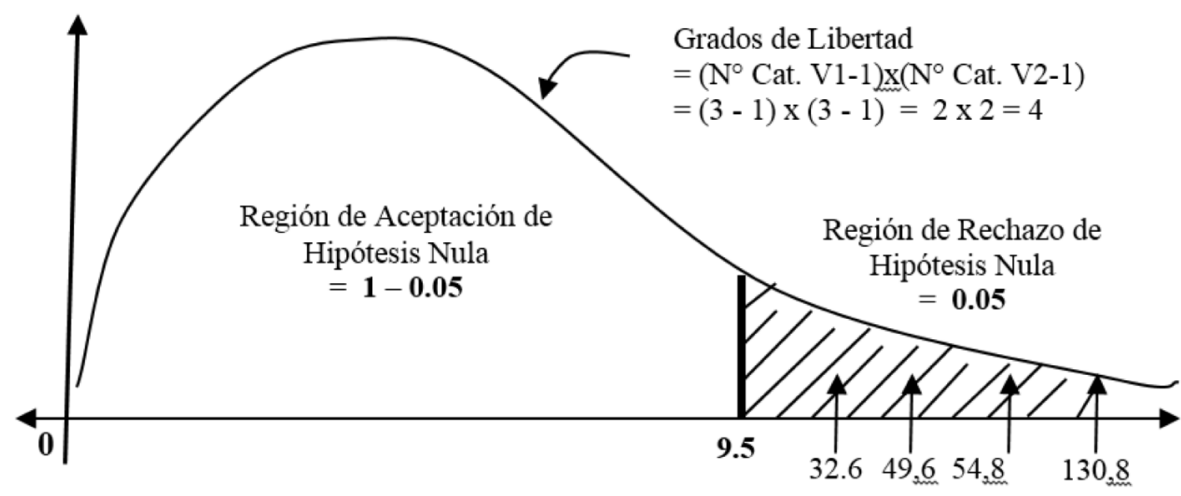

Figura 1. Contraste de hipótesis de la investigación.

Tabla 2. Resultados Inferenciales de la Investigación.

\begin{tabular}{|c|c|c|c|}
\hline \multirow{2}{*}{$\begin{array}{l}\text { Contrastación de Factores } \\
\text { Conocimientos en: }\end{array}$} & \multicolumn{3}{|c|}{ Conciencia en protección del medio ambiente } \\
\hline & $\begin{array}{l}\text { Valor Chi-cuadrado de } \\
\text { Pearson }\end{array}$ & Significancia & Interpretación \\
\hline $\begin{array}{l}\text { Negocios Basados en Valores y } \\
\text { Conciencia en Protección del Medio } \\
\text { Ambiente }\end{array}$ & 32,56 & 0,00 & $\begin{array}{l}\text { Altamente } \\
\text { significativo }\end{array}$ \\
\hline $\begin{array}{l}\text { Valores y Principios Éticos y } \\
\text { Conciencia en Protección del Medio } \\
\text { Ambiente }\end{array}$ & 130,85 & 0,00 & $\begin{array}{l}\text { Altamente } \\
\text { significativo }\end{array}$ \\
\hline $\begin{array}{l}\text { Gestión Responsable y Conciencia en } \\
\text { Protección del Medio Ambiente }\end{array}$ & 49,62 & 0,00 & $\begin{array}{l}\text { Altamente } \\
\text { significativo }\end{array}$ \\
\hline $\begin{array}{l}\text { Responsabilidad Social Empresarial y } \\
\text { Conciencia en Protección del Medio } \\
\text { Ambiente }\end{array}$ & 54,78 & 0,00 & $\begin{array}{l}\text { Altamente } \\
\text { significativo }\end{array}$ \\
\hline
\end{tabular}

\section{DISCUSIÓN}

De acuerdo con los resultados de nuestra investigación, se confirma que el nivel de conocimientos sobre responsabilidad social empresarial (RSE) si se relaciona con la protección del medio ambiente en los estudiantes de la Facultad de Ingeniería Industrial, Sistemas e Informática. Los conocimientos sobre RSE han sido analizados a través de sus factores como; negocios basados en valores, valores y principios éticos, y gestión responsable. Estos conocimientos en promedio tienen la categoría de intermedio, lo cual indica que los estudiantes tienen limitaciones en sus reflexiones para el cuidado y protección del medio ambiente. Las mejoras de los 
conocimientos y capacidades de los estudiantes universitarios de ingeniería, para promover actividades de negocios con responsabilidad social son necesarias, de esta manera se podrán egresar profesionales con alto grado de sensibilidad en el cuidado y protección del medio ambiente.

Sobre este tema la ABB en Perú (2016), en la décima edición de la expoferia de Proyectos de responsabilidad social empresarial, que organizó la asociación civil Perú 2021, ABB compartió su experiencia, visión y sus planes de responsabilidad social. Se planteó que las empresas deben planear conseguir un equilibrio entre sus resultados económicos y su intervención en la sociedad; la cual requiere un modelo de organización cuya misión, visión, estrategia y objetivos organizacionales estén directamente relacionados con el concepto de bienestar de la sociedad. Su objetivo es alcanzar el equilibrio entre la responsabilidad social y su objetivo empresarial con el fin de obtener ventaja competitiva en el futuro. Las organizaciones deben caer en la cuenta que las prácticas socialmente responsables son una herramienta para que las grandes empresas la utilicen en función de crear una ventaja competitiva.

Así mismo la Ing. Lourdes Cárdenas, Country Sustainability Controller de ABB en Perú, indicó que la compañía busca difundir, ante la comunidad, su comportamiento ético y los valores que sostienen la relación con sus colaboradores y stakeholders. "Un ejemplo de ello son las acciones orientadas a formar personas más conscientes y responsables con el cuidado del medio ambiente y la salud", aseguró. Describió los proyectos de responsabilidad social en los que participó $\mathrm{ABB}$ en Perú, entre los que destaca la promoción del Concurso de Aplicación de Eficiencia Energética, para la cual se asesoró, a más de 500 estudiantes de ingeniería de 15 universidades del país, en el uso responsable de la energía en la industria, motivándolos a presentar proyectos novedosos y eficientes para el sector empresarial, que es el gran responsable de consumo de la electricidad en el país.

En ambos casos, las pretensiones son de concientizar a las personas, sobre todo en estudiantes escolares y universitarios para desarrollar conciencia sobre la Responsabilidad Social Empresarial, intenciones que coinciden con el presente estudio también con el fin de promover conciencia sobre la RSE.

\section{REFERENCIAS BIBLIOGRÁFICAS}

ABB (28-05-2016). ABB en Perú, Apuesta por una Responsabilidad Social Compartida. Recuperado de:

http://www.abb.com.pe/cawp/seitp202 14567ef5b0e3edd09c1257b7900420b 98.aspx

Nel, L. (2010). Metodología de la Investigación, Estadística Aplicada en la Investigación. Lima, Perú. Empresa Editora Macro.Quezada, N. (2010). Metodología de la investigación: Estadística aplicada en la investigación. Lima, Perú: Empresa Editora MACRO.

Ortiz, P. (2009). La Responsabilidad Social Empresarial como Base de la Estrategia Competitiva de HZX. Tesis para optar el título de Administración de Empresas. Pontificia Universidad Javeriana. Bogotá D.C. Obtenido de: http://javeriana.edu.co/biblos/tesis/ economia/tesis242.pdf

Reyno, M. (2006). Responsabilidad Social como Ventaja Competitiva. Chile, Universidad Viña del Mar.

Hernández, R., Fernández, C. y Baptista, M. (2014). Metodología de la investigación. México D.F. Sexta Edición. Editorial McGraw-Hill.

Sánchez, H. y Reyes, C. (2017).

Metodología y Diseños en la Investigación Científica. Lima, Perú. Editorial Business Support Aneth S.R.L.

Correo electrónico: lopez2410@yahoo.es

Revisión de pares:

Recibido: 24-09-2018

Aprobado: 20-12-2018 